\title{
Hiperostosis craneal como forma de presentación de un adenocarcinoma metastásico
}

\author{
L. SUÁREZ ÁlVAREZ, N. MUELAS GÓMEZ1' J. A. TODOLÍ PARRA, T. SEVILLA \\ MANTECÓN ${ }^{1}$, J. R. CALABUIG ALBORCH \\ Servicios de Medicina Interna $y^{\prime}$ Neurología. Hospital Universitario La Fe. Valencia
}

CRANIAL HYPEROSTOSIS AS A METASTASIC ADENOCARCINOMA
PRESENTATION FORM

\begin{abstract}
RESUMEN
La hiperostosis es un aumento de masa ósea por unidad de volumen de etiología muy diversa. Presentamos el caso de una mujer de 68 años con hiperostosis craneal que debutó con clínica de protusión frontal derecha, cefalea y sintomatología neurológica. Las pruebas de imagen demostraron la existencia de hiperostosis de calota con afectación meníngea, sin lesiones cerebrales ni células malignas en líquido cefalorraquídeo. Los datos analíticos eran inespecíficos. La biopsia craneal mostró amplia infiltración neoplásica por adenocarcinoma metastásico tanto en hueso como en meninges. No se localizó el tumor primario tras realizarse TAC y mamografía.
\end{abstract}

\begin{abstract}
Hyperostosis is a volume-unit osseous increase of very diverse etiology. We present the case of a 68-year woman with a cranial hyperostosis debuting with frontal protrusion, headache and neurologic symptoms. Image proves demonstrated a hiperostosys in the calotte and meningeal inhancement, without intracerebral lesions nor malignant cells in the cerebrospinal fluid. Analytic data were unspecific. Cranial biopsy showed huge neoplastic infiltration in bone and meninges. Primary site remained unknown after a CAT and a mammography.
\end{abstract} presentación de un adenocarcinoma metastásico. An Med Interna (Madrid) 2004; 21: 548-550.

\section{INTRODUCCIÓN}

La hiperostosis se define como un aumento de masa ósea por unidad de volumen. Aunque puede responder a muy diversas causas, se observa típicamente en las zonas adyacentes a tumores o a infecciones crónicas (1). La hiperostosis craneal secundaria a infiltración neoplásica de médula ósea y duramadre es una forma infrecuente de presentación de neoplasias. Aportamos un caso en el cual la hiperostosis craneal ha sido la manifestación de debut de un adenocarcinoma metastático de primario desconocido.

\section{CASO APORTADO}

Mujer de 68 años con cefalea de un año de evolución y protusión frontal. Presentaba antecedentes de diabetes mellitus tipo 2, HTA, asma bronquial extrínseca y osteoporosis.

El cuadro comenzó con cefalea localizada en cara y hemicráneo derecho, detectándose aumento de la VSG y anemia, por lo que se inició tratamiento empírico con glucocorticoides ante la sospecha de una arteritis temporal. En aquel momento no se realizó biopsia de dicha arteria. Cuatro meses más tarde la paciente sufrió pérdida de visión bilateral. En el examen oftalmoscópico existían signos de hipertensión intracraneal. Se realizó RMN cerebral que mostró engrosamiento difuso de calota y meninges. Analíticamente persistía la anemia, con tendencia a macrocitosis. En el análisis del LCR se halló hiperproteinorraquia (seguía en tratamiento con corticoides) y no se objetivaron células malignas. Tras realizarse biopsia de la arteria temporal se descartó la existencia de arteritis y fue remitida a nuestro hospital para completar estudio.

A la clínica antes referida se asociaron crisis de sacudidas en hemicuerpo derecho que cedieron con clonazepam, alucinaciones visuales complejas, cambios en el carácter con mayor irritabilidad y pérdida de memoria reciente.

La exploración física general mostraba una paciente apirética, con palidez cutáneo-mucosa, protusión frontal derecha y con signos cutáneos de hipervascularización craneal. Presentaba edema en ambos párpados y ptosis palpebral derecha (Fig. 1). No se palpaban adenopatías. La auscultación cardiopulmonar era normal. A nivel neurológico estaba desorientada temporalmente y apática. Presentaba fatigabilidad muscular. Puntuó 19 sobre 30 en el Mini-mental test. Se objetivó disminución de agudeza visual en ambos ojos, en mayor

Trabajo aceptado: 3 de mayo de 2004

Correspondencia: Luisa Suárez Álvarez. C/ José Ma Haro, 51-13. 46022 Valencia. e-mail: Lu_sua@ hotmail.com 


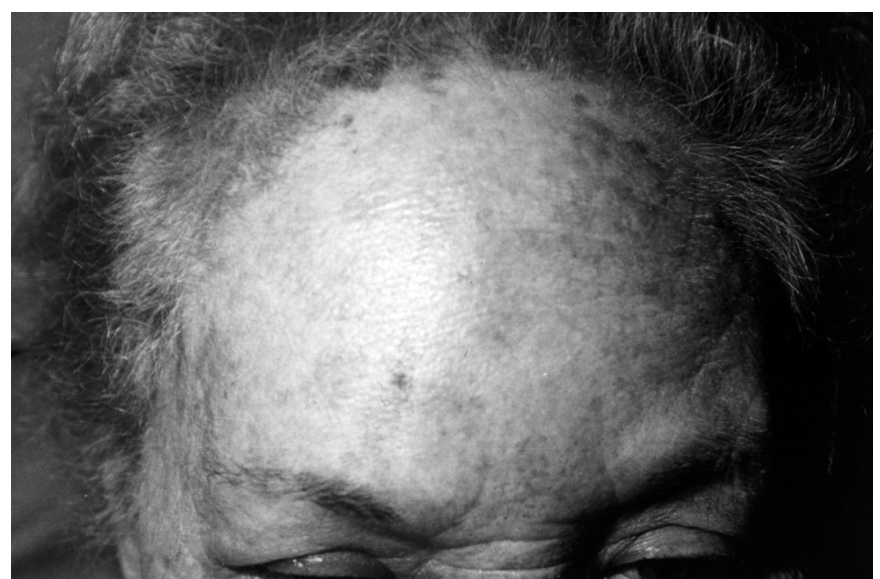

Fig. 1. Paciente con protusión ósea frontal y ptosis.

medida en el izquierdo (con el derecho contaba dedos a un metro, con el izquierdo a medio metro). En el fondo de ojo se visualizó edema de papila y exudados algodonosos bilaterales, más marcados en el ojo izquierdo. El balance motor era simétrico. Deambulaba a pasos cortos con disminución del braceo.

En la analítica sanguínea destacaba GGT 85 UI/l; LDH 779 UI/l; hipoproteinemia (proteínas totales 5,61 g/dl, albúmina 3,4 g/dl); resto de parámetros de la bioquímica dentro de la normalidad. El hemograma mostraba anemia ( $\mathrm{Hb} \mathrm{8,6} \mathrm{g/dl)} \mathrm{con} \mathrm{patrón} \mathrm{férrico} \mathrm{inflamatorio,} \mathrm{plaque-}$ tas de $106.000 / \mathrm{mm} 3$ y leucocitos normales. Hemostasia normal. VSG elevada $(53 \mathrm{~mm} / \mathrm{h}$ ). Se detectó hipotirodismo primario (TSH 1,1 mUI/l, T4 libre $0,6 \mathrm{ng} / \mathrm{dl})$ y aumento de PTH $(170 \mathrm{pg} / \mathrm{ml})$. Proteinograma y IEF sin hallazgos patológicos. Entre los marcadores tumorales sólo el CA 15,3 estaba elevado $(67,4 \mathrm{UI} / \mathrm{ml})$. La TAC craneal mostró engrosamiento de calota difuso sugestivo de hiperostosis con engrosamiento meníngeo-dural disperso más marcado en región frontal izquierda. En la punción lumbar se obtuvo un líquido cefalorraquídeo claro, con leve hiperproteinorraquia ( $48 \mathrm{mg} / \mathrm{dl}$ ), glucosa $74 \mathrm{mg} / \mathrm{dl}$ y 0 células. No se hallaron células malignas. Los cultivos del LCR fueron negativos. Se realizó un estudio EEG que mostró una lentificación difusa con actividades lentas intermitentes frontales y signos lesivo-irritativos en área temporal izquierda. La RM cerebral detectó hiperostosis difusa de calota craneal con realce meníngeo anormal difuso más prominente en región frontal izquierda (Fig. 2). La mamografía fue normal. En la serie ósea metastásica se apreció osteosclerosis generalizada. El rastreo óseo mostró refuerzo de actividad en cráneo, sobre todo a nivel fronto-parietal, compatible con hiperostosis. La biopsia de cresta iliaca presentó trabéculas artefactadas sin actividad hematopoyética. La TAC toracoabdómino-pélvica no encontró alteraciones.

Ante la presencia de una paquimeningitis con hiperostosis secundaria sin detectarse células malignas en LCR en punciones lumbares seriadas y sin conocimiento de la existencia de un cáncer primario, se realizó una biopsia de hueso craneal y meninges con el siguiente resultado anatomo-patológico: calota con hiperostosis, médula ósea y duramadre con amplia infiltración por adenocarcinoma metastático. El perfil inmunohistoquímico no orientó hacia el origen del tumor primario.

Se indicó tratamiento radioterápico craneal que la paciente rechazó, iniciándose tratamiento paliativo con tamoxifeno ante la sospecha de un tumor mamario. De hecho, unos meses después de la biopsia se realizó un nuevo TAC toraco-abdomino-pélvico que mostró un nódulo axilar derecho irregular. La paciente rechazó tratamientos agresivos y falleció en su domicilio dos meses después del alta, por lo que no se realizó estudio necrópsico.

\section{DISCUSIÓN}

La hiperostosis consiste en un aumento de la masa ósea detectable en la radiografía como una mayor densidad del
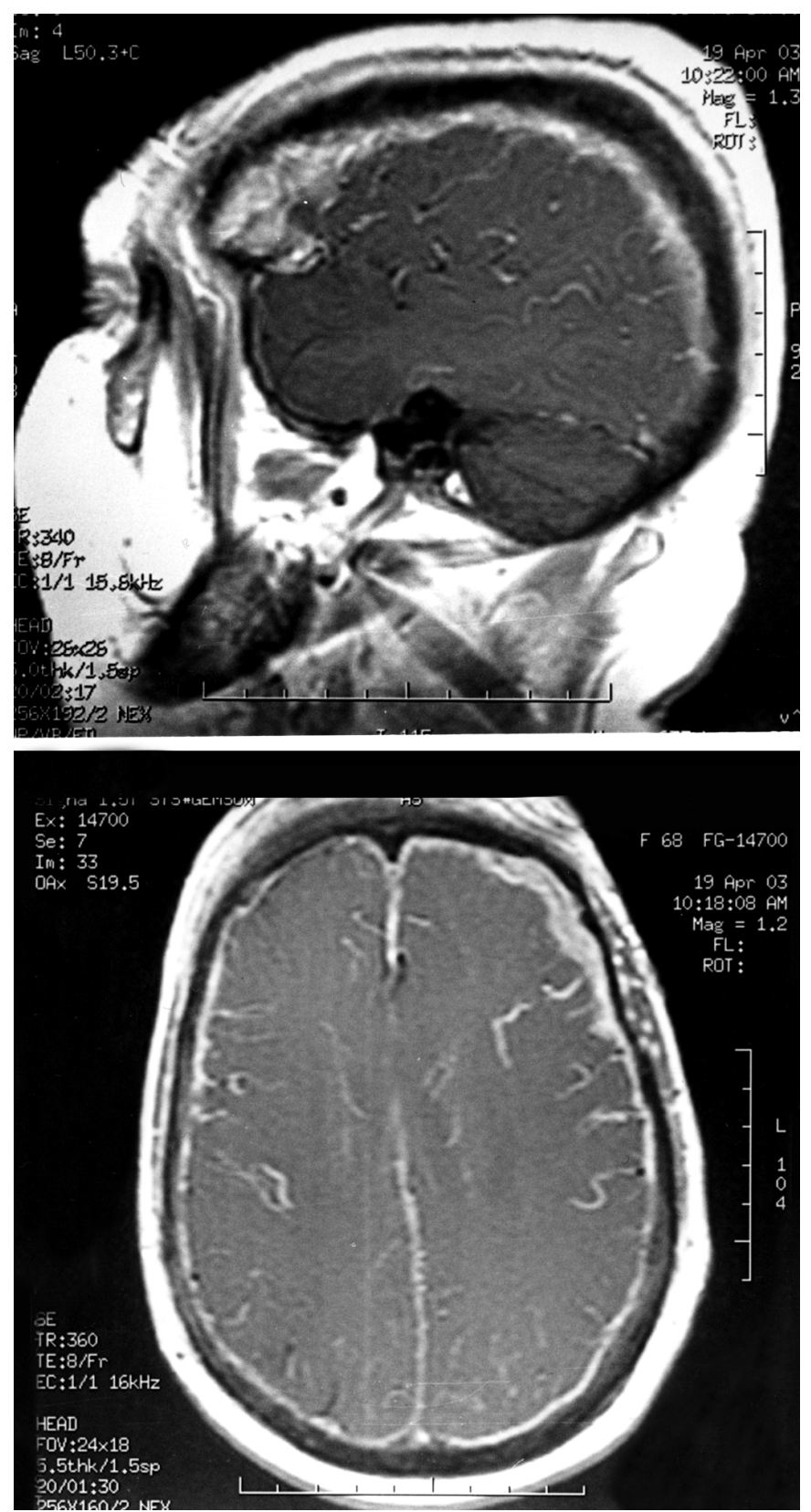

Fig. 2. RMN craneal con contrase.

hueso, generalmente asociada a un trastorno de la arquitectura tisular (1).

Los procesos a los que se asocia son diversos (2) (Tabla I)

El adenocarcinoma de localización primaria desconocida es un problema clínico frecuente. Sin embargo, la presentación como metástasis en duramadre $(3,4)$ con hiperostosis de calota es inusual. Entre las neoplasias que con mayor frecuencia metastatizan a este nivel destacan las de mama y próstata (5-7). En cuanto al cáncer de mama, suele asociarse al subtipo lobular infiltrante que tiende a metastatizar a médula ósea y desde ahí infiltra meninges, a diferencia del ductal infiltrante con predisposición a producir metástasis en parénquima cerebral $(8,9)$.

El origen del adenocarcinoma de localización primaria desconocida sólo se identifica en un $15-20 \%$ de los pacien- 


\section{TABLA I}

\section{CAUSAS DE HIPEROSTOSIS}

Trastornos endocrinos (hiperparatiroidismo, hipotiroidismo, acromegalia) Osteítis por radiación

Intoxicación química (fluoruro, fósforo, arsénico, plomo, vit A...)

Osteomalacia

Osteoesclerosis localizada asociada a infección crónica

Fase osteoesclerótica de la enfermedad de Paget

Asociada a metástasis carcinomatosas, linfoma y trastornos hematológicos

(leucemia, mieloma, drepanocitosis, mastocitosis...)

Osteopetrosis

Otras enfermedades no clasificables:

Hiperostosis frontal interna

Hiperostosis cortical generalizada

Picnodisostosis

Hiperfosfatasia

Osteomieloesclerosis

Displasia diafisaria progresiva

\section{Bibliografía}

1. Jaccobson HG. Dense bone-too much bone: Radiological consideration and differential diagnosis, part II. Skeletal Radiol 1985; 13: 97.

2. Krane SM, Schiller Al. Enfermedad de Paget y otras displasias del hueso. En: Braunwald, Fauci el al editores. Harrison. Principios de Medicina Interna. $15^{\text {a }}$ edición. Madrid: McGraw-Hill-Interamericana; 2002. p. 2619: 343.

3. Mustafa MS, Al-Nuaim L, Inayat-Ur-Rahman N. Scalp and cranial bone metastasis of endometrial carcinoma: case report and literature review. Gynecol Oncol 2001; 81: 105-9.

4. Smith DB, Howell A, Harris M, Bramwell VH, Sellwood RA. Carcinomatous meningitis associated with infiltrating lobular carcinoma of the breast. Eur J Surg Oncol. 1985; 11: 33-6.

5. Tomlin JM, Alleyne CH. Transdural metastasis from adenocarcinoma of the prostate mimicking subdural hematoma: case report. Surg Neurol. 2002; 58 (5): 329-31; discussion 331

6. Varlet, et al. Secondary subdural hematoma in dural metastasis of prostatic adenocarcinoma. Rev Neurol (Paris) 2001; 157: 1543-5.

7. Scarrow AM, Rajendran PR, Marion D. Metastatic prostate adenocarci-

tes. Las pruebas iniciales deben centrarse en determinar la extensión de la enfermedad metastática (10). La búsqueda del tumor primitivo debería limitarse a los subgrupos de pacientes en los que es posible un tratamiento específico. En metástasis únicas puede ser útil el tratamiento locoregional con cirugía o radioterapia (12). Otros pacientes sólo se beneficiarán de tratamiento sintomático y en ocasiones de quimioterapia o radioterapia paliativas (11). La TAC abdominal puede localizar el tumor primario entre un 10 a un $35 \%$ de los pacientes (13). Además se recomienda la práctica de una mamografía en mujeres y determinación del PSA en hombres. El resto de marcadores tumorales no suele ser relevante para el diagnóstico, pero con frecuencia se encuentran elevados y pueden ser de utilidad de forma seriada para evaluar la evolución y respuesta al tratamiento. En gran número de casos de adenocarcinoma que debutan con metástasis no se consigue localizar el tumor primario (80-85\%) como ocurrió con nuestra paciente $(14,15)$. noma of the dura mater. Br J Neurosurg 2000; 14: 473-4.

8. Posner, Jerome B. En: Neurologic complications of cancer. Philadelphia: F.A. Davis Company; 1995; 2 y 7; p. 32-5, 108-9, 143-63.

9. Adams RD, Victor M, Ropper AH. En: Principios de Neurología. Mc Graw-Hill Interamericana; 1999; 575-9.

10. Shildt RA, Kennedy PS, Chen TT, et al. Management of patients with metastatic adenocarcinoma of unknown origin: a Southwest Oncology Group study. Cancer Treat Rep 1983; 67: 77.

11. Pavlidis, et al. Diagnostic and therapeutic management of cancer of an unknown primary. Eur J Cancer 2003; 39: 1990-2005.

12. Fizazi K, Culine S. Metastatic carcinoma of unknown origin. Bull Cancer; 85: 609-17.

13. Karsell PR, Sheedy PO, Conell MJ. Computerized tomography in search of cancer of unknown origin. JAMA 1982; 248: 340.

14. Gil Gil M, et al. Test diagnósticos en neoplasias de origen desconocido. Estudio retrospectivo. Rev Clin Esp 1990; 186: 252-8.

15. Romeu J, et al. Carcinoma de origen desconocido. Estudio diagnóstico de 48 casos. Med Clin (Barc) 1989; 92: 201-6. 